\title{
Desarrollo sostenible en el Perú: Evidencia del periodo 1994-2015
}

\author{
Sustainable development in Perú: Evidence from the period 1994-2015
}

\author{
Carlos Enrique Orihuela Romero ${ }^{1 *}$; Rosa Nora Rojas Torres ${ }^{2}$ \\ *Autor de correspondencia
}

\section{Resumen}

El objetivo del estudio es evaluar la sostenibilidad de la economía peruana durante el periodo 1994-2015. Para ello se utilizó el criterio Inversión Genuina, bajo el cual si el valor de la base productiva ha sido no decreciente en un periodo dado (inversión genuina positiva), entonces la economía ha estado en la senda del desarrollo sostenible en ese periodo. Los resultados sugieren que la economía peruana estuvo en la senda del desarrollo sostenible durante el periodo en análisis, aunque ello no garantiza necesariamente que la base productiva siga creciendo endógenamente, y por ende, la capacidad para enfrentar el desarrollo futuro.

Palabras clave: desarrollo sostenible; riqueza; inversión genuina; pib; ingreso.

\begin{abstract}
The purpose of this study is to evaluate the sustainability of Peruvian economy during 1994-2015 using the Genuine Investment criterion, which states that if the value of the productive base has been non-decreasing in a given period, then the economy has been on the sustainable development path. The results suggest that the Peruvian economy was on the sustainable development path in the period under analysis, although this does not necessarily guarantee that productive base will continue to grow endogenously, and therefore, the capacity to face the future development.
\end{abstract}

Keywords: sustainable development; wealth; genuine investment; gdp; income.

\section{Introducción}

Si bien la tasa de crecimiento del producto interno bruto (PIB) per-cápita es vista como el indicador clásico de crecimiento económico, no necesariamente es el más apropiado para evaluar el desempeño de una economía. Cuando un país rico en recursos naturales no renovables extrae tales recursos (por ejemplo, metales), eventualmente incrementa su dinámica económica y PIB, pero al mismo tiempo reduce su stock de capital natural, pudiendo mermar su capacidad para generar ingresos en el futuro (incluso a pesar de los nuevos descubrimientos). Así, el PIB no permite inferir mucho sobre el futuro. Por ello, parte de la literatura económica (Dasgupta, 2011; Word Bank, 2003; 2006; 2011) está interesada en evaluar el desarrollo sostenible como alternativa.

Evaluar el desarrollo sostenible de una economía es fundamental para los países altamente dependientes de la venta de sus recursos naturales, en donde los bajos niveles de reinversión de rentas pueden poner en peligro la satisfacción de necesidades de las generaciones futuras. Por ello, Dasgupta y Mäler (2001) propusieron el indicador Inversión Genuina como el más apropiado para evaluar si una economía ha estado en la senda del desarrollo sostenible durante un horizonte dado. Este indicador mide el cambio del valor de la base productiva o riqueza de una economía durante un periodo dado. Si una economía acumula riqueza, entonces tendrá la capacidad para satisfacer las necesidades futuras, de manera que estaría en capacidad de alcanzar su nivel de desarrollo deseado o sostenible.

El objetivo del presente estudio es determinar si un país rico en recursos naturales, pero con bajos niveles de PIB per-cápita como el Perú puede mantener sus posibilidades de desarrollo en el futuro. En otras palabras, emplear el indicador Inversión Genuina para evaluar la sostenibilidad de la economía peruana. La presunción es que el Perú ha estado en la senda del desarrollo sostenible durante el periodo 1994-2015 de la mano con el crecimiento económico experimentado en gran parte de ese periodo.

Las contribuciones de Lange (2004), Atkinson y Gundimeda (2006), Arrow et al. (2007), Kumar (2013), Ollivier y Giraud (2011) para evaluar el desarrollo sostenible Botswana-Namibia, India, Estados UnidosChina, Mozambique e India, respectivamente, refuerzan el consenso en la literatura económica para utilizar este indicador.

Asimismo, esta investigación propone mejorar los resultados de la evaluación de la riqueza peruana por el World Bank (2006, 2011). Para ello se propone el uso de: i) información

\footnotetext{
${ }^{1}$ Facultad de Economía y Planificación, Universidad Nacional Agraria La Molina, Lima-Perú. E-mail: corihuela@lamolina.edu.pe ${ }^{2}$ Facultad de Economía y Planificación, Universidad Nacional Agraria La Molina, Lima-Perú. E-mail: 20111033@lamolina.edu.pe
} 
proveniente de fuentes locales en lugar de fuentes externas; ii) estimaciones de costos marginales de extracción de recursos naturales; iii) valorar el capital humano usando información de retornos a la educación, iv) ampliar el horizonte de estudio. Los resultados de este estudio deberían ser más precisos.

Para el desarrollo del estudio, en la sección dos se comenta la metodología, la cual incluye el marco teórico enfatizando en los conceptos básicos para este estudio y un breve análisis de estudios similares. En la sección tres se presentan los resultados mientras que en la sección cuatro se señalan las conclusiones, limitaciones y recomendaciones.

\section{Metodología}

\subsection{Marco conceptual}

Dasgupta y Mäler (2001) proponen el indicador Inversión Genuina (I) como el más apropiado para evaluar el desarrollo sostenible de una economía. Ellos sostienen que una senda de desarrollo es sostenible si el bienestar social es no decreciente en toda la senda. El modelo parte de la función de bienestar social (V), la cual se define como el valor presente de la utilidad (agregada o social) a lo largo de la senda. Así, V equivale a un bienestar intergeneracional. Koopmans $(1960,1965)$ demostró que, bajo condiciones generales, V debería ser entendido como:

$$
\mathrm{V}_{\mathrm{t}}=\sum_{\mathrm{s}=\mathrm{t}}^{\infty} \frac{\mathrm{U}_{\mathrm{s}}}{(1+\delta)^{s-\mathrm{t}}}
$$

donde d es la tasa de descuento de la utilidad. ${ }^{3}$ Como U no es observable, Samuelson (1948) propuso que la riqueza actual (W) debería ser igual al valor presente del consumo futuro (2): lo que se consumirá en el futuro dependerá del nivel de riqueza actual. En otras palabras, el bienestar futuro estará determinado por el nivel actual de riqueza.

$$
\mathrm{W}_{\mathrm{t}} \approx \mathrm{V}_{\mathrm{t}}=\sum_{\mathrm{s}=\mathrm{t}}^{\infty} \frac{\mathrm{U}\left(\mathrm{C}_{\mathrm{s}}\right)}{(1+\delta)^{\mathrm{s}-\mathrm{t}}}
$$

siendo $\mathrm{C}_{\mathrm{s}}$ el consumo del periodo s y $\mathrm{U}(\mathrm{C})$ la utilidad de ese consumo, la cual contiene todo lo que afecta al bienestar en un periodo dado. Con esta predicción es factible pronosticar el bienestar social:

$$
\mathrm{V}_{\mathrm{t}}=\sum_{\mathrm{t}}^{\infty} \frac{\mathrm{U}\left(\propto\left(\mathrm{s}, \mathrm{t}, \mathrm{K}_{\mathrm{t}}\right)\right)}{(1+\delta)^{\mathrm{s}-\mathrm{t}}}
$$

Obviamente, $\mathrm{V}$ es una función del stock de capital inicial $\mathrm{y}$ de su mecanismo de asignación. El patrón de consumo (y el bienestar intertemporal) depende de la evolución de la base productiva de la economía, de manera que en algún momento la producción generada por esta base es asignada entre consumo e inversión en diferentes formas de capital.

Tal asignación está determinada por dos tipos de mecanismos: autónomo y no-autónomo. Una pequeña economía abierta

${ }^{3}$ Para la convergencia de $\mathrm{V}$ se requiere que $\mathrm{d}>0$. El lector puede revisar Hepburn (2007) y Dasgupta (2001) quienes ofrecen una excelente discusión al respecto. generalmente tiene un mecanismo de asignación noautónomo ya que sus decisiones dependen también de factores externos. Asumiendo un mecanismo de asignación no-autónomo, V será una función explicita del tiempo y para el caso de un solo tipo de capital, $\mathrm{K}$ :

$\mathrm{V}_{\mathrm{t}}=\mathrm{V}(\mathrm{K}(\mathrm{t}), \mathrm{t})$

Calculando el diferencial total de (4):

$\mathrm{dV}_{\mathrm{t}}=\frac{\partial \mathrm{V}}{\partial \mathrm{K}} \mathrm{dK}+\frac{\partial \mathrm{V}}{\partial \mathrm{t}} \mathrm{dt}$

Aplicando la derivada total con respecto a t, (5) puede ser re-expresado como:

$$
\frac{\mathrm{dV}}{\mathrm{dt}}=\mathrm{p}_{\mathrm{t}} \frac{\mathrm{dK}}{\mathrm{dt}}+\frac{\partial \mathrm{V}}{\partial \mathrm{t}}
$$

Donde $p_{t}=\partial \mathrm{V} / \partial \mathrm{K}$. El miembro del lado izquierdo de la expresión (6) equivale a la inversión genuina $(\mathrm{I}=\mathrm{dV} / \mathrm{dt})$ la cual se define como la variación del bienestar intertemporal, variación de la riqueza o cambio en la base productiva. $\mathrm{Si}$ esta variación es no decreciente en el tiempo entonces la generación futura tendría al menos las mismas oportunidades para generar bienestar tal como las tuvo la generación predecesora.

De esta forma, si $I_{t}>0$ entonces la riqueza aumento en el periodo t. En otras palabras, la economía se encontró en la senda sostenible durante el periodo señalado, puesto que generó los recursos (en valor social) necesarios para satisfacer el desarrollo futuro. Caso contrario, la economía no se encontró en la senda sostenible en el periodo $t$.

El primer miembro del lado derecho de la expresión (6) representa la variación del capital $\mathrm{K}$ valorado por su respectivo precio cuenta constante, $\mathrm{p}_{\mathrm{t}}$. Este precio se define como el valor presente de los futuros beneficios netos de un capital resultante de una perturbación en su stock inicial o como la contribución de una unidad adicional de capital al bienestar social (7). Entonces, la correcta estimación de este precio sombra es clave (UNU-IHDP y UNEP, 2012).

$\mathrm{p}_{\mathrm{t}}=\frac{\partial \mathrm{V}_{\mathrm{t}}}{\partial \mathrm{K}_{\mathrm{t}}}=\sum_{\mathrm{t}}^{\infty} \frac{\left[\frac{\partial \mathrm{U}\left(\alpha\left(\mathrm{s}, \mathrm{t}, \mathrm{K}_{\mathrm{t}}\right)\right)}{(1+\delta)^{s-t}}\right]}{(1+\delta)^{s-t}}$

El segundo miembro () es conocido como el "drift term" el cual es independiente de la base productiva y representa el efecto de los cambios exógenos tales como cambios tecnológicos e institucionales, productividad y bienes públicos globales. Es usual estimarlo mediante el Factor de Productividad Total (TFP) o residual de Solow. Nótese que el TFP es en realidad un tipo de capital. Extendiendo (6) en tiempo discreto y para el caso de tres tipos de capital: manufacturado $(\mathrm{K})$, natural $(\mathrm{N})$ y humano $(\mathrm{H})$ se obtiene $(8)$ :

$I_{t}=\Delta W=\sum_{i}\left(p_{\text {Kit }} \frac{\mathrm{dK}_{\mathrm{it}}}{\mathrm{dt}}\right)+\sum_{\mathrm{i}}\left(\mathrm{p}_{\mathrm{Njt}} \frac{\mathrm{dN}_{\mathrm{jt}}}{\mathrm{dt}}\right)+\sum_{\mathrm{m}}\left(\mathrm{p}_{\mathrm{Hmt}} \frac{\mathrm{dH}}{\mathrm{dt}}\right)$

En este caso, la inversión genuina será equivalente a la sumatoria de las variaciones de los diversos tipos de capital valorados por su precio cuenta. Para un periodo t, $\mathrm{K}_{\mathrm{it}}$ es la 
cantidad del i-ésimo capital manufacturado, $\mathrm{N}_{\mathrm{jt}}$ la cantidad de la j-ésima forma de capital natural y $\mathrm{H}_{\mathrm{mt}}$ el m-ésimo tipo de capital humano, siendo sus respectivos precios sombra $\mathrm{p}_{\mathrm{Ki}}, \mathrm{p}_{\mathrm{NJ}}, \mathrm{y} \mathrm{p}_{\mathrm{HM}}$.

Operacionalmente, la inversión genuina puede evaluarse también mediante la evolución de la riqueza medida a precios sombra constantes (9).

$$
\mathrm{W}=\sum_{\mathrm{i}} \mathrm{p}_{\mathrm{Kit}_{\mathrm{it}}} \mathrm{dK}_{\mathrm{it}}+\sum_{\mathrm{j}} \mathrm{p}_{\mathrm{Njt}} \mathrm{dN}_{\mathrm{jt}}+\sum_{\mathrm{m}} \mathrm{p}_{\mathrm{Hmt}} \mathrm{dH}_{\mathrm{mt}}
$$

Cuando la tasa de crecimiento de la población es significativa y exógena entonces debería calcularse el valor del cambio del capital per- cápita (10), el cual depende del ratio capital/ población (L) y de las tasas de crecimiento del capital y de la población. Debe resaltarse que (10) no equivale a una inversión genuina per-cápita.

$\mathrm{I}_{\mathrm{t}}=\mathrm{p} \Delta\left(\frac{\mathrm{K}}{\mathrm{L}}\right)=\mathrm{p} \frac{\mathrm{K}}{\mathrm{L}}\left[\frac{\Delta \mathrm{K}}{\mathrm{K}}-\frac{\Delta \mathrm{L}}{\mathrm{L}}\right]$

Nótese que este criterio no asume que el Estado maximiza el bienestar social ni requiere optimizar los recursos o capital, ya que simplemente se basa en el pronóstico o mapeo del capital inicial hacia otro conjunto de programas económicos. Asimismo, aunque la degradación ambiental no es considerada explícitamente en el modelo, lo es implícitamente. Es de esperar que esta degradación incida en los activos de la economía y por ende debería ser capturada en el precio sombra y por ende, en su valor social (riqueza).

Dado que la I utiliza información ex-post, simplemente evalúa si una economía ha estado en la senda hacia el desarrollo sostenible. En otras palabras, el indicador no garantiza que la economía haya presentado un desarrollo sostenible: simplemente indica si la economía ha estado acumulando (o reduciendo) su base productiva para enfrentar un futuro desarrollo que pueda ser sostenible. Una economía que presentó magros indicadores de salud, educación e incluso de instituciones, puede haber logrado una inversión genuina positiva. No obstante, surge una paradoja: esa economía puede estar en la senda del desarrollo sostenible aun cuando sus indicadores sociales sugieran la ausencia de algún tipo de desarrollo.

En realidad, no hay condición suficiente que garantice la sostenibilidad futura. Es imposible que exista un indicador cuya predicción sea perfecta. Lo que hay son condiciones que garantizan que la economía estuvo o no en la senda del desarrollo sostenible en un periodo dado. Sin embargo, es posible inferir si la economía potencialmente puede mantenerse en tal senda.

Utilizar el cambio en la riqueza hace que la inversión genuina sea operacionalmente factible. Sin embargo, se requiere que los precios sombra sean estimados adecuadamente (Newmayer, 2013; Martinet, 2012). De esta forma, la estimación del precio sombra constituye una especie de "Talón de Aquiles" de la inversión genuina (Smulders, 2012). Al margen de todo, quizá la fortaleza más significativa de la inversión genuina es proveer mucha información para los analistas y/o tomadores de decisión sobre los cambios en los activos identificados y por lo tanto, proveer una guía para futuras inversiones (UNU-IHDP y UNEP, 2012).

\section{Inversión Genuina y Ahorro Genuino ${ }^{4}$}

El Ahorro Genuino (AG) también es un indicador basado en riqueza y ha sido propuesto por el World Bank (2006; 2011) para evaluar el desarrollo sostenible de todos sus países miembros. Las diferencias -en comparación al indicador Inversión Genuina- radican tanto en el marco conceptual como en la parte operacional.

El AG se basa en la expresión (11), en la cual asume que la riqueza es el flujo descontado del consumo. La formulación asume que el consumo se encuentra sobre una senda sostenible. Esto implica que los ahorros serán suficientes para compensar la depreciación del capital. Se calculan tres tipos de valores de capital: natural, humano e intangible, siendo este último obtenido como un residual, luego de estimar el valor del capital total.

En el caso de la inversión genuina, no hay supuestos sobre la sostenibilidad del consumo: los cambios en la riqueza son obtenidos directamente a partir de cambios en el valor de los activos. Mientras que la I utiliza (8) para estimar el cambio en la riqueza en un periodo $t$, el AG lo hace a partir del (11), siendo e la base de logaritmo neperiano y $r$ la tasa social de retorno de la inversión.

$W_{t}=\int_{t}^{\infty} \mathrm{C}(s) \cdot \mathrm{e}^{-\mathrm{t}(\mathrm{s}-\mathrm{t})} \mathrm{ds}$

Operativamente, mientras la I requiere información de los stocks de capital de la economía (de un periodo dado) y sus respectivos precios cuenta, el AG actualiza (valor presente) los hipotéticos niveles de consumo proyectados hacia un periodo predeterminado.

En la literatura, cuando la riqueza es obtenida mediante (11) se denomina comprehensive wealth, mientras que si es estimada a partir de (10) será inclusive wealth. Hasta donde se conoce, no hay traducciones en la literatura de estas expresiones, las cuales podrían interpretarse como: patrimonio y riqueza inclusiva, respectivamente.

Es evidente que la I posee mayores ventajas sobre el AG no solo desde el punto de vista operacional sino también desde una perspectiva teórica: i) no requiere asumir si el consumo se encuentra en una senda sostenible; ii) tampoco asume que los ahorros futuros serán suficientes para compensar la depreciación que debería ocurrir en el futuro. La I puede generar trayectorias no sostenibles lo cual permite resaltar los tipos de capital que están reduciéndose y por ende, proponer las medidas correctivas.

Diversos estudios han estimado el AG e I para diversas economías (Tabla 1). Quizá el estudio más conocido fue el realizado por el World Bank (2011) para todos sus países miembros. En base a supuestos heroicos de este estudio,

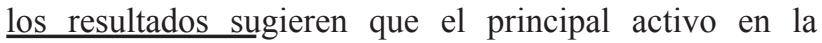
${ }^{4}$ También llamado "comprehensive wealth" y "net savings". 
economía mundial es el denominado "intangible", el cual es obtenido como un residual y equivale básicamente al capital institucional.

\subsection{Aplicación al caso peruano}

A efectos de calcular la Inversión Genuina para el Perú durante el periodo 1994-2015 se utilizarán las principales formas de capital: i) artificial $\left(\mathrm{K}^{\mathrm{F}}\right)$, ii) natural $\left(\mathrm{K}^{\mathrm{N}}\right)$, el que comprende minería (oro, plata, cobre, zinc, plomo, hierro, y estaño), hidrocarburos (petróleo, gas natural, líquidos

Tabla 1. Estudios de Inversión Genuina y Ahorro Genuino

\begin{tabular}{|c|c|c|c|}
\hline Autor & $\begin{array}{l}\text { Indicador } \\
\text { utilizado }\end{array}$ & Periodo & $\begin{array}{c}\text { Lugar de } \\
\text { aplicación }\end{array}$ \\
\hline Arrow et al (2012) & I & $2000-2005$ & $\begin{array}{l}\text { Estados } \\
\text { Unidos, China, } \\
\text { Brasil, India, y } \\
\text { Venezuela }\end{array}$ \\
\hline UNU-IHDP y UNEP (2012) & I & 1990-1998 & 20 países \\
\hline World Bank (2011) & $\mathrm{AG}$ & $\begin{array}{c}1995,2000 \\
2005\end{array}$ & $\begin{array}{l}\text { Países que } \\
\text { forman parte del } \\
\text { World Bank }\end{array}$ \\
\hline Ferreira y Moro (2011) & $\mathrm{I}$ & $1995-2005$ & Irlanda \\
\hline Ollivier y Giraud (2011) & $\mathrm{AG}$ & $2000-2005$ & Mozambique \\
\hline Walker et al (2010) & I & 1991-2001 & Australia \\
\hline Mota et al (2010) & $\mathrm{AG}$ & $1990-2005$ & Portugal \\
\hline Arrow et al (2007) & I & $2000-2005$ & $\begin{array}{l}\text { Estados Unidos y } \\
\text { China }\end{array}$ \\
\hline Lange (2004) & I & 1980-1998 & $\begin{array}{l}\text { Botswana } \\
\text { Namibia }\end{array}$ \\
\hline
\end{tabular}

IG: inversión genuina; AG: ahorro genuino

de gas natural), y pesca de anchoveta, y iii) humano $\left(\mathrm{K}^{\mathrm{H}}\right)$. Formalmente:

$\mathrm{I}_{\mathrm{t}}=\mathrm{p}_{\mathrm{t}}^{\mathrm{F}} \Delta \mathrm{K}_{\mathrm{t}}^{\mathrm{F}}+\mathrm{p}_{\mathrm{t}}^{\mathrm{N}} \Delta \mathrm{K}_{\mathrm{t}}^{\mathrm{N}}+\mathrm{p}_{\mathrm{t}}^{\mathrm{H}} \Delta \mathrm{K}_{\mathrm{t}}^{\mathrm{H}}+\mathrm{v}_{\mathrm{t}}$

Donde:

I : Inversión genuina

$\mathrm{p}^{\mathrm{F}} \quad$ : Precio cuenta del capital artificial

$\mathrm{p}^{\mathrm{N}} \quad$ : Precio cuenta del capital natural

$\mathrm{p}^{\mathrm{H}} \quad$ : Precio cuenta del capital humano

$\mathrm{v} \quad$ : Drift term (residual)

Nótese que se utiliza precio cuenta en lugar de precio sombra. Mientras que el primero es un precio teórico y extremadamente difícil de calcular, el segundo es una aproximación que se basa en el beneficio marginal de consumir o producir un bien o servicio. A continuación, se detalla la forma de estimación de las cuentas físicas y monetarias de cada tipo de capital.

\section{Capital Artificial}

El primer término de (8), equivale al stock de capital artificial del país $\left(\mathrm{K}^{\mathrm{F}}\right)$, el cual es estimado utilizando el Método Inventario Perpetuo (MIP), siendo relativamente sencillo de implementar, puesto que solo requiere información de la inversión en precios constantes (I), horizonte de vida de los activos (n) y tasa anual de depreciación $(\alpha)$. Formalmente
(13):

$\mathrm{K}_{\mathrm{t}}^{\mathrm{F}}=\sum_{\mathrm{i}=0}^{\mathrm{n}} \mathrm{I}_{\mathrm{t}-\mathrm{i}}(1-\propto)$

Los resultados $\mathrm{K}_{t}^{\mathrm{F}}$ para la economía peruana fueron obtenidos de Seminario (2012) quien utilizó el MIP para estimar el periodo 1994-2011. El periodo 2012-2015 fue extrapolado. Dado que el precio sombra de este capital es equivalente a la unidad, lo que se calcula entonces es . $p_{t}^{F}$ $K_{t}^{F}$

\section{Capital Natural}

El capital natural esta a su vez conformado por numerosos sub-tipos de capital, siendo los más conocidos minería, petróleo, gas natural, bosques, pesquerías, suelo agrícola, entre otros. UNU-IHDP y UNEP (2012) sugieren la inclusión de otras formas de capital natural en el cálculo de la riqueza, como ecosistemas y recursos hídricos, no obstante dificultad en la medición de ambas constituye una gran limitación para tal inclusión. El caso peruano no es la excepción.

Para priorizar los tipos de capital natural se tomó en cuenta la información disponible y la contribución del capital natural al PIB. Según este criterio, suelo agropecuario $\left(\mathrm{K}^{\mathrm{A}}\right)$, minero $\left(\mathrm{K}^{\mathrm{M}}\right)$, hidrocarburos $\left(\mathrm{K}^{\mathrm{H}}\right)$ y pesquero $\left(\mathrm{K}^{\mathrm{P}}\right)$ fueron considerados como los más relevantes. No se incluyó el sector forestal, puesto que su contribución al PIB es mínima ${ }^{5}$ y tampoco hay información disponible que permita calcular precios cuenta ni la evolución de sus stocks. A continuación, se detalla la forma de estimación tanto de las cuentas físicas como monetarias según el tipo de capital natural.

\section{Suelo agropecuario}

El sector agropecuario comprende básicamente, la actividad agrícola y pecuaria, las cuales representan el grueso del valor bruto de la producción sectorial. El aporte de la silvicultura es mínimo y por ende no será analizado en este estudio. Por ello, se asume que el capital suelo agropecuario $\left(\mathrm{K}^{\mathrm{A}}\right)$ comprende básicamente el capital suelo agrícola $\left(\mathrm{K}^{\mathrm{AG}}\right)$ y el capital suelo pecuario $\left(\mathrm{K}^{\mathrm{AP}}\right)$, de manera manera $\mathrm{K}_{t}^{\mathrm{A}}=\mathrm{K}_{\mathrm{t}}{ }^{\mathrm{AG}}+\mathrm{K}_{\mathrm{t}}{ }_{\mathrm{AP}}$. Puesto que en el Perú solo se han realizado censos agropecuarios los años 1994 y 2012 (INEI, 2013a; INEI y MINAG, 2013), solo existe información física agropecuaria para ambos años. Con la

${ }_{5}^{5}$ El Ministerio de Agricultura no ofrece información de la evolución de los stocks maderables ni costos de extracción. En las cuentas nacionales, el valor agregado de la actividad forestal está incluida en el valor agregado agropecuario puesto que la contribución de la primera es mínima, la cual -según algunas estimaciones- equivale a menos del 1\% del PIB total. Por ende, es muy difícil calcular el valor del cambio del capital forestal, motivo por el cual no ha sido considerado en el presente estudio. La escaza participación del sector forestal en el PIB total tiene que ver en cierta forma con la extracción ilegal cuyo producto no logra ser registrado en las cuentas nacionales. 
tasa de crecimiento implícita entre los años 1994 y 2012 se estimó la superficie para todo el periodo en análisis.

El precio cuenta del sector agropecuario no está disponible y tuvo que ser estimado. Este precio puede ser estimado mediante la diferencia del precio de mercado y el costo marginal de producción, incluyendo externalidades positivas y negativas.

El sector agrícola peruano comprende numerosos cultivos. $\mathrm{Si}$ bien los precios de mercado de todos los productos agrícolas están disponibles, los costos de producción no lo están. Por ello se decidió utilizar el excedente de explotación agropecuario $\left(\mathrm{EE}^{\mathrm{A}}\right)$ como una aproximación del beneficio bruto agropecuario. Esto ahorra la tarea de estimar el beneficio para cada cultivo.

Para descomponer este excedente en el excedente de explotación agrícola $\left(\mathrm{EE}^{\mathrm{AG}}\right)$ y excedente de explotación pecuario $\left(\mathrm{EE}^{\mathrm{AP}}\right)$, se requiere los factores de contribución del sector agrícola y pecuario $(\alpha$ y $(1-\alpha)$, respectivamente) al excedente de explotación agropecuario. Formalmente, (14):

$$
\mathrm{EE}_{\mathrm{t}}^{\mathrm{A}}=\alpha\left(\mathrm{EE}_{\mathrm{t}}^{\mathrm{AG}}\right)+(1-\alpha)\left(\mathrm{EE}_{\mathrm{t}}^{\mathrm{AP}}\right)
$$

La estimación de todas estas variables es detallada en Orihuela (2015). Una vez estimados los excedentes agrícola y pecuario, se procede a calcular los correspondientes precios cuenta, (15) y (16). Nótese que los precios cuenta -dada la forma de estimación propuesta- equivalen a beneficios promedio y no beneficios marginales. Estos precios corresponden al año 1994.

$$
\mathrm{P}_{\mathrm{t}}^{\mathrm{AG}}=\alpha \frac{\mathrm{EE}_{\mathrm{t}}^{\mathrm{A}}}{\mathrm{K}_{\mathrm{t}}^{\mathrm{AG}}}
$$

$$
\mathrm{P}_{\mathrm{t}}^{\mathrm{AP}}=(1-\alpha) \frac{\mathrm{EE}_{\mathrm{t}}^{\mathrm{A}}}{\boldsymbol{K}_{\mathrm{t}}^{\mathrm{AP}}}
$$

No hay estudios a nivel local que hayan evaluado los retornos de escala del sector agrícola y/o pecuario. Conforme a INEI y MINAG (2013), la mayor parte de las unidades de producción agropecuarias en el Perú son relativamente pequeñas (menores a 5 ha) cuyo número ha sido creciente en el periodo de estudio, lo cual sugiere un fraccionamiento cada vez mayor de estas unidades.

En este contexto, parece razonable asumir la presencia de retornos constantes a escala en el sector agropecuario peruano. Así, estimar el precio cuenta como un beneficio promedio (en lugar de beneficio marginal) no debería generar distorsiones significativas en los resultados.

Una vez obtenidos los dos precios cuenta, la riqueza del suelo agropecuario $\left(\mathrm{W}^{\mathrm{A}}\right)$ será equivalente a la sumatoria del capital suelo agrícola y suelo pecuario valorados por sus respectivos precios cuenta (en este caso, $\mathrm{P}^{\mathrm{AG}}$ y $\mathrm{P}^{\mathrm{AP}}$ ). Formalmente (17):

$$
\mathrm{W}_{\mathrm{t}}^{\mathrm{A}}=\mathrm{P}_{\mathrm{t}}^{\mathrm{AG}} \mathrm{K}_{\mathrm{t}}^{\mathrm{AG}}+\mathrm{P}_{\mathrm{t}}^{\mathrm{AP}} \mathrm{K}_{\mathrm{t}}^{\mathrm{AP}}
$$

\section{Capital Minero}

El sector minero peruano es polimetálico de manera que comprende la extracción, concentración y refinación (según el metal) de oro, plata, cobre, plomo, zinc, hierro y estaño, los cuales constituyen los principales metales comerciales, y que a su vez representan el grueso de los ingresos y valor agregado sectorial.

Mediante una función de costos translog, Orihuela (2015) estimo el costo marginal de la extracción de todos los metales en análisis para el periodo 1994-2010. Con esta informacion se estimó el precio cuenta por metal.

Las estadísticas de reservas probadas por metal fueron obtenidas del Ministerio de Energía y Minas - MINEM (varios años).

\section{Capital pesquero}

Las principales especies pesqueras comerciales del Perú son la anchoveta y sardina, las cuales han concentrado el grueso de los ingresos sectoriales. Solo la anchoveta ha concentrado el $78-92 \%$ de la extracción total durante el periodo 2005-2011 (INEI 2013b). Por ello, su extracción es representativa de la pesquería peruana.

El precio cuenta de la pesquería peruana tuvo que ser estimado a partir de la información de las cuentas nacionales. Puntualmente, como la división del excedente de explotación pesquero extractivo entre la cantidad extraída o desembarque de anchoveta. La información del $\mathrm{EE}^{\mathrm{P}}$ estuvo disponible en los compendios estadísticos del INEI mientras que la estadística de desembarque total del sector pesquero fue obtenida de PRODUCE e INEI (2013b).

La información de biomasa de anchoveta está disponible para todo el periodo de estudio (INEI, 2013b). Esta biomasa equivale al capital pesquero $\left(\mathrm{K}^{\mathrm{P}}\right)$.

\section{Capital hidrocarburos}

En el Perú, la mayor parte del valor bruto de la producción del sector hidrocarburos está conformado por la extracción y venta de petróleo crudo, gas natural y líquidos de gas natural (LGN). Estos últimos tomaron fuerza a partir de la implementación del proyecto Camisea. El Perú es tomador de precios dado que la extracción hidrocarburos es marginal con respecto al total mundial. Las estadísticas de reservas probadas de petróleo, gas natural y LGN están disponibles en el MINEM (2013b).

Para el caso del petróleo crudo, Ego-Aguirre (2012) estimó la función de costo total de la industria petrolera peruana para el periodo 1996-2010 en la zona costera y selva, en base a la cual el autor estimó no solo los costos marginales de extracción por zona sino también el precio cuenta respectivo. Esta información fue utilizada en este estudio.

No hay información sobre precios cuenta de gas natural y LGN en el Perú. Ambos hidrocarburos han sido extraídos en pequeñas cantidades y de forma conjunta con el petróleo hasta el inicio del Proyecto Camisea (año 2004). Desde 
entonces fueron extraídos como productos principales. Este proyecto concentra la mayor parte de la extracción de ambos hidrocarburos.

En este caso, no fue posible construir una función de costo total multi-producto debido al escaso número de observaciones, puesto que el Proyecto Camisea empezó sus operaciones el año 2004. Además, la empresa extrae no solo gas natural y LGN, sino otros líquidos de natural, lo cual dificultaría un análisis desagregado de costos por producto.

Para superar esto, inicialmente se optó por estimar el precio de venta de cada uno de los dos principales productos de la empresa (gas natural y LGN) en base a la información financiera de la empresa. Si bien la empresa ofrece información de costos, no fue posible separarlos para cada uno de los dos productos a fin de calcular un beneficio marginal. En base a la rentabilidad de la empresa, definida como beneficio operativo/ingresos totales por venta, se infirió el beneficio (privado) de cada uno de los productos señalados.

\section{Capital humano}

La literatura ofrece al menos dos métodos para valorar el capital humano: valor de una vida estadística (VVE) y método del capital humano (MCH). El primero (VVE) consiste en medir la disposición a pagar por evitar una muerte. El VVE es calculado dividiendo la disposición de pago marginal para reducir el riesgo de muerte por el tamaño de la reducción de riesgo. Este método ofrece el valor más alto de un daño a la salud. El segundo método $(\mathrm{MCH})$ estima el costo indirecto de productividad perdida mediante el valor de los ingresos futuros que serían perdidos.

Desde una perspectiva microeconómica, el VVE constituiría el método correcto puesto que captura las preferencias de la sociedad por reducir el riesgo de muerte, e indirectamente, por la vida humana. En el Perú no hay estudios o estimaciones sobre la disposición de pago por reducir el riesgo en mención. Si bien este dato puedo extrapolarse de otros países (mediante el método de transferencia de beneficios), se incurría en una distorsión al introducir las preferencias de una sociedad en otra.

Quizá por este motivo, aunado a la disponibilidad de información que se requiere y a la sencillez de su aplicación, es que el valor del capital humano ha sido frecuentemente estimado mediante el MCH. Los estudios más conocidos sobre inversión genuina y ahorro genuino, tales como Arrow et al (2012) y World Bank (2011) utilizaron este método. Así, el método tiene la ventaja de que los resultados sean comparables con trabajos similares.

Orihuela (2015) utiliza el MCH para calcular el stock de capital humano peruano y el precio sombra respectivo para el periodo 1994-2012, cifras que fueron actualizadas al año 2015.

\section{Drif Term}

El último término del lado derecho de la expresión (12), es el drift term, el cual equivale a la contribución a la inversión genuina que no es atribuible a los capitales considerados explícitamente en el modelo, como, por ejemplo, capital institucional, ecosistemas, etc. Dado que es difícil el estimar tal contribución, normalmente se utiliza la tasa de crecimiento del TFP como una aproximación al drift term.

A partir de Orihuela y Nolazco (2013), los autores actualizaron sus resultados para el periodo 1994-2012, encontrando que contribución acumulada del TFP en el periodo de análisis fue $47 \%$. Se reconoce que, a partir de este resultado, sería difícil llegar a la contribución exacta del capital institucional no solo al cambio del producto sino también al cambio de la riqueza. Dada las limitaciones de información, se utilizará este resultado (47\%) como una mejor aproximación en lugar del TFP convencional en el periodo en análisis.

\section{Capital Financiero}

Los activos externos netos representan los activos -propiedad de los agentes nacionales privados, empresas, y agencias gubernamentales- que son mantenidos en otros países. Estos activos pueden representar una importante fuente de ingresos en economías que presentan bajos niveles de inversión doméstica (Lange, 2004). Estos activos constituyen una forma de capital artificial. Si bien estos activos no forman parte explícita del modelo, también constituyen una fuente de riqueza de una economía y por ende serán incluidos en la expresión (12). La información de estos activos está disponible en el BCRP (2013).

Todas las cifras monetarias fueron convertidas a soles constantes 1994 utilizando el deflactor implícito del PIB. Esta información fue obtenida del INEI.

\section{Resultados}

De los resultados (Tabla 2) se desprende que la mayor fuente de riqueza recayó largamente en el capital humano $(60 \%)$ mientras que el segundo lugar recayó en el capital artificial (26\%). Estos capitales constituyen la base para sostener todas las actividades económicas.

El capital natural más significativo ha sido el capital minero, aunque con apenas 9\% de la riqueza total. Si bien el suelo agropecuario constituye un capital importante, su aporte a la riqueza ha sido poco significativo. Dadas las amplias diferencias entre los diferentes tipos de riqueza, cálculos más finos para los diferentes tipos de capital natural no debería cambiar significativamente los resultados. ${ }^{6}$

La riqueza total aumentó durante el periodo en análisis (171\%), es decir la economía peruana ha estado acumulando capital (en términos monetarios) de manera que su base productiva se ha incrementado sustancialmente. En otras

${ }^{6}$ Nótese que el capital suelo agropecuario concentra apenas ha concentrado un $1 \%$ de la riqueza total, de manera que la eventual sobrestimación en su cálculo es irrelevante. 
palabras, la inversión genuina es positiva. Esto sugiere -a priori- que la economía peruana ha estado en la senda del desarrollo sostenible.

Más aun, los resultados van en línea con los obtenidos por World Bank (2006; 2011); Hamilton y Atkinson (2006) en donde la riqueza en diversos bloques de países (años 2000 y 2005) se concentró en el capital intangible (equivalente al capital humano y capital institucional), argumentándose que el desarrollo económico mundial estuvo basado en una rápida acumulación (monetaria) del capital artificial e intangible a costa de un excesivo agotamiento del capital natural. Esto no fue el caso peruano, en donde hubo acumulación en la riqueza de todos los capitales que conforman la base productiva.

La contribución de la riqueza natural con respecto a la riqueza total se ha reducido (Tabla 3 ). La riqueza humana (capital artificial, capital humano y activos externos netos) es la que más ha aumentado en términos absolutos y relativos, mientras que la riqueza artificial ha estado rezagada en términos relativos. Nótese que se redujo la participación de la riqueza natural.

La riqueza per-cápita también aumentó sustancialmente durante el periodo en análisis (140\%), incluso a una tasa superior al crecimiento del PIB per-cápita (102\%) (Tabla $4)$.

Tabla2. Riqueza peruana según tipo de capital (millones de nuevos soles 1994)

\begin{tabular}{|c|c|c|c|c|c|c|c|c|}
\hline Año & Artificial & $\begin{array}{c}\text { Activos } \\
\text { Externos } \\
\text { Netos }\end{array}$ & Humano & $\begin{array}{c}\text { Suelo } \\
\text { Agropecuario }\end{array}$ & Minero & Hidrocarburos & Pesca & Total \\
\hline 1994 & 302,713 & 13,057 & 451,028 & 8,801 & 89,187 & 14,197 & 1,575 & 880,558 \\
\hline 1995 & 315,322 & 13,777 & 428,625 & ,912 & 104,394 & 13,320 & 819 & 885,169 \\
\hline 1996 & 328,900 & 18,660 & 467,378 & 9,0 & 104,914 & 12,388 & 910 & 942,168 \\
\hline 1997 & 343,239 & 16,136 & 490,435 & 9,1 & 117,886 & 11,787 & 1,119 & 989,729 \\
\hline 1998 & 358,449 & 15,813 & 640,240 & 9,239 & 150,290 & 12,983 & 441 & $1,187,455$ \\
\hline 1999 & 371,234 & 18,534 & 741,066 & 9,351 & 158,072 & 11,325 & 655 & $1,310,236$ \\
\hline 2000 & 382,573 & 17,485 & 698,150 & 9,464 & 144,535 & 11,815 & 572 & $1,264,595$ \\
\hline 2001 & 391,924 & 18,971 & 791,069 & 9 & 179,0 & 14, & 1,306 & $1,406,451$ \\
\hline 2002 & 399,426 & 22,598 & 895 & 9,6 & 173 & & 867 & $1,515,135$ \\
\hline 2003 & 408,068 & 22,880 & 932,774 & 9,8 & 175 & & 907 & $1,562,998$ \\
\hline 2004 & 417,276 & 25,150 & 960,011 & 9,9 & 147, & 13,8 & 1,318 & $1,574,935$ \\
\hline 2005 & 427 & 28,425 & 1,20 & & & & 1,483 & 1,81 \\
\hline 2006 & 442,755 & 31,164 & $1,268,295$ & 10,1 & 141,548 & 15,205 & 935 & $1,910,080$ \\
\hline 2007 & 465,340 & 43,149 & $1,333,475$ & 10,303 & 192,951 & 16,342 & 963 & $2,062,523$ \\
\hline 2008 & 476,640 & 52,653 & $1,373,616$ & 10,430 & 158,221 & 19,420 & 1,272 & $2,092,252$ \\
\hline 2009 & 510,958 & 50,958 & $1,399,896$ & 10,5 & 148,603 & 19 & 951 & $2,141,277$ \\
\hline 2010 & 553,495 & 62,817 & $1,367,346$ & 10,688 & 171,516 & 21,196 & 947 & $2,188,004$ \\
\hline 2011 & 598,991 & 63,386 & $1,394,772$ & 10,820 & 176,519 & 21,103 & 1,225 & $2,266,815$ \\
\hline 2012 & 555,744 & 74,990 & $1,408,351$ & 10,953 & 129,843 & 23,092 & 1,084 & $2,204,057$ \\
\hline 2013 & 570,414 & 86,445 & $1,421,229$ & 11,089 & 220,421 & 26,985 & 1,431 & $2,338,015$ \\
\hline 2014 & 585,084 & 83,110 & $1,431,493$ & 11,226 & 246,880 & 24,869 & 791 & $2,383,454$ \\
\hline 2015 & 599,755 & 90,609 & $1,442,554$ & 11,365 & 223,600 & 17,313 & 1,225 & $2,386,422$ \\
\hline $\mathrm{TC}(\%)$ & 98 & 594 & 220 & 29 & 151 & 22 & -22 & 171 \\
\hline Riqueza (\%) & 26 & 2 & 60 & 1 & 9 & 1 & 0 & 100 \\
\hline
\end{tabular}

* Periodo 1994-2015

** El valor promedio de un capital es dividido entre el valor promedio de la riqueza total

Tabla 3. Contribución (\%) en la riqueza según capital 1994 y 2015

\begin{tabular}{cccccccc}
\hline Año & Artificial & $\begin{array}{c}\text { Activos } \\
\text { Externos Netos }\end{array}$ & Humano & $\begin{array}{c}\text { Suelo } \\
\text { Agropecuario }\end{array}$ & Minero Hidrocarburos & Pesca \\
\hline 1994 & 34.4 & 1.5 & 51.2 & 1.0 & 10.1 & 1.6 & 0.2 \\
2015 & 25.1 & 3.8 & 60.4 & 0.5 & 9.4 & 0.7 & 0.1 \\
\hline
\end{tabular}

Tabla 4. Tasas de crecimiento (\%) de la riqueza per-cápita, ajustado por TFP durante el periodo 1994-2015

\begin{tabular}{|c|c|c|c|c|c|}
\hline $\begin{array}{l}\text { Tasa de } \\
\text { crecimiento de } \\
\text { la riqueza }\end{array}$ & $\begin{array}{c}\text { Tasa de } \\
\text { crecimiento de la } \\
\text { población* }\end{array}$ & $\begin{array}{l}\text { Tasa de } \\
\text { crecimiento de la } \\
\text { riqueza per-cápita }\end{array}$ & $\begin{array}{l}\text { Tasa de } \\
\text { ecimiento del } \\
\text { TFP }\end{array}$ & $\begin{array}{l}\text { Tasa de crecimiento } \\
\text { de la riqueza per- } \\
\text { cápita incorporando } \\
\text { TFP }\end{array}$ & $\begin{array}{c}\text { Tasa de } \\
\text { crecimiento del } \\
\text { PBI per-cápita }\end{array}$ \\
\hline (1) & (2) & $(3)=(1)-(2)$ & (4) & $(5)=(3)+(4)$ & (6) \\
\hline 171 & 31 & 140 & 47 & 187 & 102 \\
\hline
\end{tabular}


Incorporando además el TFP (cambio tecnológico, capital institucional, etc.), los resultados siguen siendo positivos y alentadores. Además, altas tasas de crecimiento del ingreso requieren al mismo tiempo niveles de acumulación significativa de la riqueza. Esto ha ocurrido en la economía peruana. Esto sugiere -a priori- que la economía peruana mantiene intactas las capacidades para poder generar bienestar humano en el futuro, sin embargo, no hay garantía que los altos niveles de crecimiento (ya sea en términos per-cápita de riqueza o $\mathrm{PIB}$ ) registrados durante el periodo 1994-2015 sean mantenidos, incluso en un futuro cercano.

El creciente capital artificial podría facilitar la creación y/o crecimiento de otros capitales. La necesidad insatisfecha en el stock de capital artificial (Armendariz et al, 2011; Perroti y Sanchez, 2011) podría justificar mayores inversiones futuras en este rubro, lo cual debería generar una mayor dinámica económica (ingresos). En otras palabras, dadas las enormes carencias de infraestructura ${ }^{7}$, la economía peruana tiene el potencial para crecer endógenamente y eventualmente, satisfacer las necesidades que el desarrollo requerirá.

Considerando que el sector minero-metálico ha sido el motor de la economía peruana durante los últimos años (debido a los altos precios de los comodities y a la mayor producción metálica de ¿?) parecería que la economía local dependería en mayor medida del sector externo (exportaciones de metales) que de las inversiones en infraestructura. Esto va en línea con los hallazgos de Aparicio et al (2011) y MEF (2012), los cuales señalan la gran dependencia del crecimiento del PIB con respecto al sector externo.

Si el sector minero es y será el responsable de empujar la economía en el futuro cercano, entonces la economía todavía posee el potencial (en términos de capital natural) para financiar su desarrollo futuro y permanecer en la senda del desarrollo. Sin embargo, ello dependerá de la futura asignación de los recursos, lo cual recae no solo en la economía en sí, sino en el agente que la regula: el Estado (capital institucional) aunque en gran medida, de factores externos.

\section{Conclusiones y recomendaciones}

La inversión genuina peruana ha sido positiva durante el periodo 1994-2015. Esto corrobora la hipótesis del estudio, en el sentido que la economía del Perú estuvo en la senda del desarrollo sostenible en ese periodo. Los resultados de este estudio están limitados en diversos aspectos, básicamente a los supuestos utilizados en el análisis y al vacío de información disponible en el momento de realizar los cálculos.

El modelo "inversión genuina" no garantiza si una economía o sociedad ha logrado algún tipo de desarrollo. Tampoco si habiendo alcanzado la senda del desarrollo sostenible, este pueda mantenerse en el futuro. Por ende,

${ }^{7}$ Se estima que el déficit seria US\$ 80000 millones, de los cuales la mitad debería recaer en el sector transporte y comunicaciones (Semana Económica, 2014). una inversión genuina positiva no permite asegurar la sostenibilidad futura de la economía.

Para lograr el desarrollo sostenible o permanecer en su senda, la sociedad peruana debería implementar medidas para la mejora/aumento de capitales, principalmente el capital artificial, capital articulador de la economía, y el capital institucional, el cual asigna o redistribuye los recursos públicos en la sociedad. Futuros estudios deberían evaluar la cartera de actividades a implementar. Esto es crucial para un país extremadamente dependiente de la extracción y venta de recursos naturales

\section{Literatura citada}

Aparicio, C.; Aragon, G.; Rodríguez, J. 2011. ¿Qué factores explican las fluctuaciones recientes del producto bruto interno peruano?: Un análisis a través de un Modelo de Equilibrio General. Documento de trabajo. Superintendencia de Banca, Seguros y Administradoras Privadas de Fondos de Pensiones.

Armendariz, E.; Jaramillo, F.: Zegarra, L. 2011. Barreras al crecimiento económico de Junín. Diagnóstico y Propuesta 48, Consorcio de Investigación Económica y Social (CIES). Banco Interamericano de Desarrollo.

Arrow, K.; Dasgupta, P.; Goulder, L.; Mumford, K.; Oleson, K. 2007. China, the U.S., and Sustainability: Perspectives Based on comprehensive Wealth. Working Paper No. 313. Stanford Center for International Development. Stanford University.

Arrow, K.; Dasgupta, P.; Goulder, L.; Mumford, K.; Oleson, K. 2012. Sustainability and the measurement of wealth. Environment and Development Economics, 17(3): 317-353.

Atkinson, G. y Gundimeda, H. 2006. Accounting for India's Forest Wealth. Ecological Economics, 59 (4): 462-476.

BCRP 2013: Memoria 2012. Banco Central de Reserva del Perú.

Dasgupta, P. 2001. Human Well-being and the Natural Environment. Oxford University Press. 351p.

Dasgupta, P. and Mäler, K-G. 2001. Wealth as a Criterion for Sustainable Development. World Economics, 2(3): $19-44$.

Ego-Aguirre, M. 2012. Economías de escala del sector petrolero peruano, periodo 1996-2010. Tesis de Maestría, Programa Economía de los Recursos Naturales y del Ambiente. Universidad Nacional Agraria La Molina, Escuela de Posgrado.

Hamilton, K. and Atkinson, G. 2006. Wealth, Welfare and Sustainability. Advances in Measuring Sustainable Development. Edward Elgar. Chetelham, UK. 201p.

Hepburn, C. 2007. Valuing the far-off future: discounting and its alternatives. En Handbook of Sustainable Development. Editado por Giles Atkinson, Simon Dietz y Eric Neumayer. Edward Elgar. Chetelham, UK. 489p. 
INEI. 2013a. III Censo Nacional Agropecuario 1994: Disponible en: http://www.inei.gob.pe/BancoCuadros/ bancua06a.asp?PARAMETRO=03000000Nivel:Nacio nal. Fecha de actualización: 25/09/2013.

INEI. 2013b. Compendio Estadístico del Perú 2013: Tomo No 1. Instituto Nacional de Estadística e Informática. Disponible en: http://www.inei.gob.pe/.

INEI y MINAG. 2013. IV Censo Nacional Agropecuario 2012-Resultados Definitivos. Instituto Nacional de Estadística e Informática / Ministerio de Agricultura y Riego. 62 p.

Koopmans, T. C. 1960. Stationary ordinal utility and impatience. Econometrica, (28): 287-309.

Koopmans, T. C. 1965. On the concept of optimal economic growth. Pontificae Academiae Scientiarium Scripta Varia, (28): 225-300.

Kumar, S. 2013. Comprehensive wealth and sustainable development in India. Munich Personal RePEc Archive. Disponible en: http://mpra.ub.uni-muenchen. de/43809/1/MPRA paper 43809.pdf.

Lange, G-M. 2004. Wealth, Natural Capital, and Sustainable Development: Contrasting Examples from Botswana and Namibia. Environmental and Resource Economics, 29: 257-283.

Martinet, V. 2012. Economic Theory and Sustainable Development: what can we preserve for future generations. Routledge Studies in Ecological Economics.

MEF 2012. Avances en la Agenda del MEF. Exposición del Ministro de Economía y Finanzas Luis Miguel Castilla, Abril 2012.

MINAM 2014. Agenda Ambiental del Perú 2013-2014. Disponible en: http://www.minam.gob.pe/wp-content/ uploads/2013/06/agendambiental_peru_2013-20141. pdf.

MINEM (2013b). Anuario Estadístico de Hidrocarburos 2012. Ministerio de Energía y Minas.

Mota, R.,Domingos, T., Martins, V. (2010). Analysis of genuine savings and potential green net national income: Portugal 1990-2005. Ecological Economics, 69: 1934-1942

Newmayer, E. 2013. Weak versus Strong Sustainability: Exploring the Limits of Two Opposing Paradingms, $3^{\text {rd }}$ Edition. Edward Elgar, Cheltenham.

Ollivier, T., Giraud, P. 2011. Assessing sustainability, a comprehensive wealth accounting prospect: An application to Mozambique. Ecological Economics, 70: 503-512.

Orihuela, C. 2013. Incluyendo el agotamiento de los recursos naturales en las cuentas nacionales: evidencia peruana del periodo 1994-2011. Documento preparado para la Cooperación Técnica Belga. Universidad Nacional Agraria La Molina. Documento no publicado.

Orihuela, C. 2015. ¿Estuvo Perú en la senda del desarrollo sostenible? Una aplicación basada en la sostenibilidad débil: Evidencia del periodo 1994-2012. Tesis de Doctorado, Programa Doctoral Economía de los Recursos Naturales y el Desarrollo Sustentable. Universidad Nacional Autónoma de México.

Orihuela, C. y Nolazco, J. 2013. Incorporando la Productividad Total de Factores incorporando variables ambientales: el caso peruano.Natura@economia, (2): 29-48.

Perroti, D. y Sánchez, R. 2011. La brecha en infraestructura en América Latina y el Caribe. Serie Recursos Naturales e Infraestructura 153. CEPAL.

Pezzey, J. y Toman, M. 2005. Sustainability and its economic interpretations, in R. David Simpson, Michael A. Toman and Robert U. Ayres (ed.), Scarcity and Growth Revisited, Resources for the Future Press, Washington, DC, USA, pp. 121-141.

Samuelson, Paul A. 1948. "The Business Cycle" en Economics, EUA: McGrawHill, primera edición, cap. 17.

Seminario, B. 2012. Perú: Stock de Capital, PIB potencial y Productividad. Disponible en: https://sites.google. com/site/lbseminario/peru-2021

Smulders, S. 2012. An arrow in the Achilles' hell of sustainability and the wealth accounting. Environment and Development Economics, 17: 368-372.

UNU-IHDP y UNEP. 2012. Inclusive Wealth Report 2012. Measuring progress toward sustainability. Cambridge: Cambridge University Press.

Walker, B.; Pearson, L.; Harris, M.; Mäler, K-G.; Li, C-Z.; Biggs, R. y Baynes, T. 2010. Incorporating Resilience in the assessment of Inclusive Wealth: An example from South East Australia. Environmental and Resource Economics, 45: 183-202.

World Bank. 2003. World Development Report 2003: Sustainable Development in a Dynamic World: Transforming Institutions, Growth, and Quality of Life. Washington DC. Disponible en: http:// wdronline.worldbank.org/worldbank/a/c.html/world development_report_2003/abstract/WB.0-8213-51508.abstract. Fecha: 20/10/2013.

World Bank. 2006. Where is the Wealth of the Nations? Measuring Capital for the 21st Century. Washington DC. USA.

World Bank. 2011. The changing wealth of nations. Washington, DC: World Bank. 